\title{
EVALUACIÓN DE OSTEOPOROSIS Y ATEROESCLEROSIS CAROTÍDEA EN RELACIÓN A ENFERMEDAD CEREBROVASCULAR OCLUSIVA
}

\author{
Drs. Gretel Santana R, Lesly Solís A, Ariel Gonzáles L.
}

Especialistas de 1er grado en Imagenología del Hospital Universitario "Dr. Carlos J Finlay", Ciudad de La Habana, Cuba.

\begin{abstract}
ASSESSMENT OF CORRELATION BETWEEN OCCLUSIVE CEREBROVASCULAR DISEASE AND OSTEOPOROSIS AND CAROTID ATHEROSCLEROSIS
\end{abstract}

\begin{abstract}
Objective. To determine the association between carotid atherosclerotic manifestations and osteoporosis in patients with occlusive cerebrovascular disease (OCVD). Method. From June 2007 to June 2009115 patients with clinical tomographic diagnosis of ECVO, who underwent carotid artery-Color Doppler Ultrasound exams as well as lumbar spine and hip bone densitometry, were studied. Results. No correlation between the value of bone mineral density (BMD) and the magnitude of carotid atherosclerotic damage was observed. There was a moderate positive correlation between Body Mass Index (BMI) and BMD. Most risk factors were associated with increased intima media thickness, increased atherogenic index, low rates of significant stenosis, and densitometric osteopenia, with aging and hypertension as predominant factors. Conclusions. Despite the presence of shared risk factors, no correlation between osteoporosis and atherosclerotic manifestations in the carotid artery in OCVD was observed.

Key words: Carotid atherosclerosis, Densitometry, Doppler ultrasound carotid, Occlusive cerebrovascular desease, Osteoporosis.
\end{abstract}

Resumen: Objetivo. Determinar la asociación existente entre las manifestaciones ateroescleróticas carotídeas

Santana G y cols. Evaluación de osteoporosis y ateroesclerosis carotídea en relación a enfermedad cerebrovascular oclusiva. Rev Chil Radiol 2010: 16(3); 136-146.

Correspondencia: Dra. Gretel Santana $R$.

Calle 411\% 192 y 194, \#19213, Santiago de las Vegas, Boyeros, La Habana, Cuba.

vicky@ida.cu

Trabajo recibido el 10 de febrero de 2010, aceptado para publicación el 20 de julio de 2010. y la osteoporosis, en pacientes con enfermedad cerebrovascular oclusiva (ECVO). Método. Estudiamos 115 pacientes con diagnóstico clínico tomográfico de ECVO desde junio 2007 a junio 2009, a los que se les realizó ultrasonido Doppler color carotídeo y densitometría de columna lumbosacra y caderas. Resultados: No hallamos correlación entre el valor de la densidad mineral ósea (DMO) y la magnitud del daño aterosclerótico carotídeo; encontramos correlación moderada positiva entre el índice de masa corporal y la DMO. La mayoría de los factores de riesgo se asociaron con incremento del grosor íntima media, índice aterogénico aumentado y baja prevalencia de estenosis significativa, así como osteopenia densitométrica, siendo el envejecimiento y la hipertensión los factores predominantes. Conclusiones: La osteoporosis y las manifestaciones ateroescleróticas carotídeas en la ECVO no guardan relación, más allá de la presencia de factores de riesgo en común.

Palabras clave: Ateroesclerosis carotídea, Ecodoppler carotídeo, Enfermedad cerebrovascular oclusiva, Densitometría, Osteoporosis.

\section{Introducción}

La ateroesclerosis y la osteoporosis son dos de las enfermedades crónicas más prevalentes del mundo; originan la mayor parte de la morbimortalidad en adultos mayores y su prevalencia sigue en aumento(1). Los estudios que confirman la relación entre ellas se basan en el aumento, tanto de la mortalidad global como por esta enfermedad, específicamente en personas con disminución de la masa ósea (MO). La disminución de MO también se ha asociado con el riesgo de ictus ${ }^{(2-5)}$. La enfermedad cerebrovascular (ECV) constituye la primera causa de invalidez y la tercera causa de muerte de la población adulta en los países desarrollados y en Cuba, sólo precedida por las enfermedades coronarias y el cáncer ${ }^{(6-8)}$.

Dado que la osteoporosis podría ser un marcador de riesgo de mortalidad por enfermedad ateroescle- 
rótica y específicamente por ECV, nos motivamos a realizar esta investigación que tuvo como propósito determinar la asociación existente entre las manifestaciones ateroescleróticas carotídeas y los valores cuantitativos de la osteoporosis, evaluados mediante ecografría Doppler y densitometría respectivamente, en pacientes con ECVO. Nos planteamos como pregunta para la investigación: ¿Es posible asociar las diferentes manifestaciones ateroescleróticas carotídeas con los valores cuantitativos de la DMO?

\section{Material y métodos}

Se realizó un estudio prospectivo, descriptivo y transversal en 115 pacientes que ingresaron en el Servicio de Neurología del Hospital Universitario "Dr. Carlos J. Finlay" con diagnóstico clínico y tomográfico de ECVO, en el período comprendido entre junio 2007 y junio 2009. Se determinaron los factores de riesgo ateroesclerótico: HTA, edad mayor de 60 años, diabetes mellitus (DM), cardiopatía isquémica, ECV anterior, tabaquismo, alcoholismo y dislipidemia. Las alteraciones carotídeas se estudiaron mediante ecografía Doppler color de dichas arterias y se efectuó densitometría a nivel de columna lumbosacra y caderas para determinar la DMO, en cada uno de los pacientes.

Se excluyó los siguientes pacientes:

$\checkmark$ Pacientes a los que no se les pudo realizar la ecografía Doppler de manera satisfactoria por distintos motivos, siendo los más importantes:

calcificaciones extensas que impidan evaluar el sector, cuello corto, obesidad y bifurcaciones carotídeas altas.

$\diamond$ Pacientes con diagnóstico tomográfico de hemorragia intraparenquimatosa.

Para definir el diagnóstico etiológico de la ECV isquémica nos basamos en la clasificación de los infartos cerebrales en sus diferentes subtipos etiológicos adaptada del Laussane Stroke Registry, (Bogousslavsky 1997) y del comité ad hoc del Grupo de Estudio de Enfermedades Cerebrovasculares (Arboix et al 1998). Posteriormente se evaluaron las arterias carótidas, mediante Doppler duplex color. La exploración fue siempre realizada por el mismo operador (especialista en imaginología con una experiencia de 12 años en la realización de este tipo de estudio).

Para realizar el examen ultrasonográfico se empleó un equipo marca Aloka modelo Prosound alfa 5 con un transductor lineal multifrecuencial de 7,5 a $13 \mathrm{MHz}$. El estudio se hizo en una habitación con luz atenuada para lograr una mejor apreciación de los detalles, con el paciente en decúbito supino sobre la camilla, cuello en extensión ligeramente rotado y ambos brazos en supinación, pegados al cuerpo para conseguir que los hombros descendieran el máximo posible. El transductor lineal se ubicó a cada lado del cuello del paciente, previa aplicación de gel transmisor, explorándose ambos ejes carotídeos y siguiendo siempre la secuencia que se muestra a continuación:

1. Barrido en escala de grises, con el transductor en posición transversal desde el origen de la arteria carótida común hasta la sección más distal posible de la carótida externa y la carótida interna. Exploración bilateral, comenzando siempre por el mismo lado.

2. Barrido en escala de grises, con el transductor en posición longitudinal de los mismos sectores. Para la identificación correcta de la carótida común, carótida externa y carótida interna, la exploración se realizó colocando el transductor por anterior o bien por detrás del músculo esternocleidomastoideo.

3. Medición del grosor de la íntima y media de la carótida (GIM).

4. Introducción del Doppler color.

5. Observación cuidadosa de la morfología de las placas (en caso de haberlas).

Con posterioridad se efectuó el análisis e interpretación de las imágenes, considerando los siguientes parámetros:

- Grosor carotídeo íntima-media: Para medir el GIM, se amplía el haz ultrasónico perpendicular a la arteria carótida para discriminar claramente dos líneas, una correspondiente a la interfaz íntima-sangre y la otra a la interfaz media-adventicia. EI GIM se mide como la distancia máxima entre esas dos líneas. Las determinaciones se realizaron en la carótida común a $1 \mathrm{~cm}$ del bulbo (Izcovich ED et al., 2004), cuantificando en $\mathrm{mm}$. Se tomó como parámetro patológico cifras por encima de $1.0 \mathrm{~mm}$. La validación se realizó tomando como mínimo 3 medidas y posteriomente obteniendo el promedio (Figura 1).

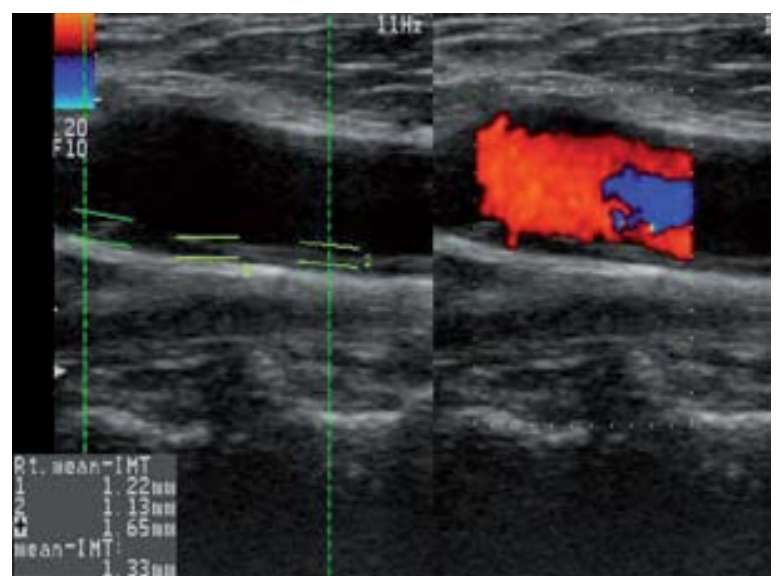

Figura 1. Técnica sistemática para la medición. 1a. Grosor intima media; promedio de 3 medidas. 


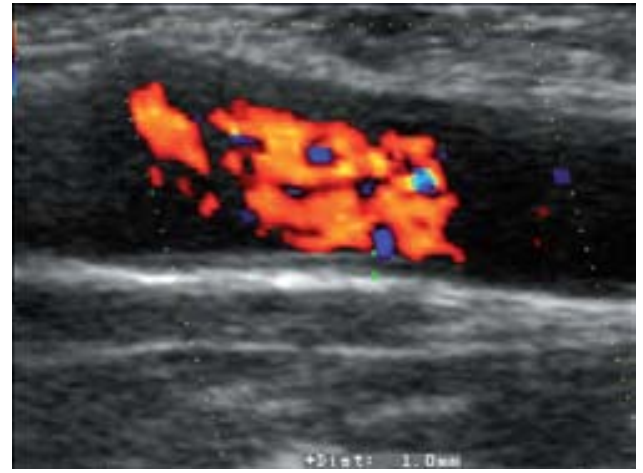

Figura 1b. Valor normal.

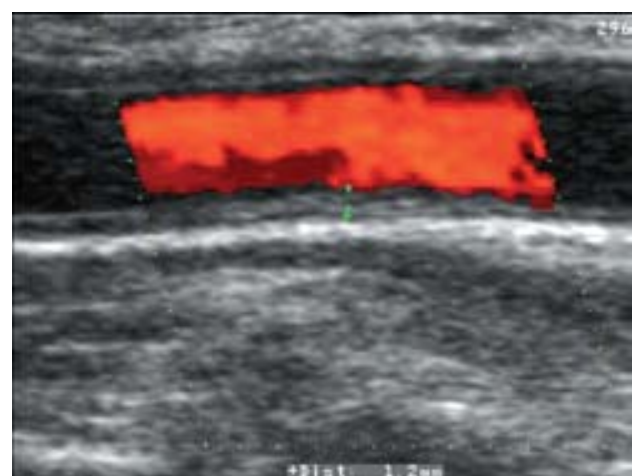

Figura 1c. Valor patológico.

- Clasificación de la placa carotídea (Consenso de Estenosis Carotídea, Sociedad Argentina de Cardiología, Sociedad Neurológica Argentina. Revista Argentina de Cardiología / vol $74 \mathrm{n}^{\circ} 2$ / marzo-abril 2006) (Figura 2).

- Uniformemente anecoica o ecolúcida (placa tipo I).

- Predominantemente hipoecogénica (placa tipo II) (>50\% hipoecogénica).

- Predominantemente hiperecogénica (placa tipo III) (> 50\% isoecogénica o hiperecogénica).

- Uniformemente isoecogénica o hiperecogénica (placa tipo IV).

- No clasificable (placa tipo V) (placas cálcicas).

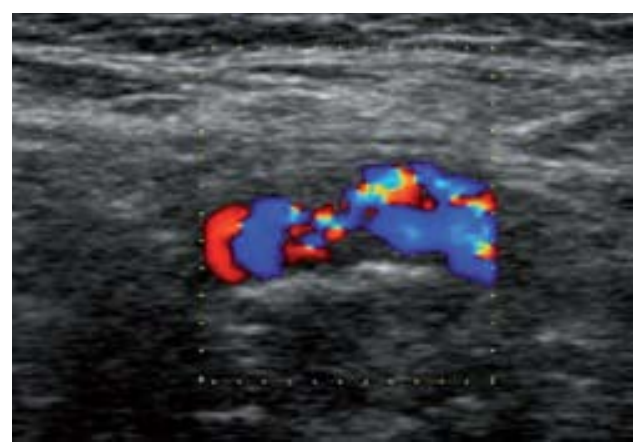

Figura 2. Tipos de placas de ateroma según su ecogenicidad. 2a. Placa tipo I. Placa ecolúcida o anecoica.

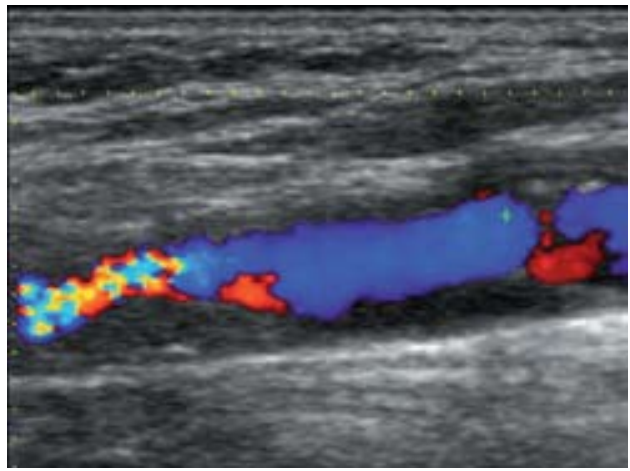

Figura 2b. Placa tipo II. Placa heterogénea predominantemente hipoecoica.

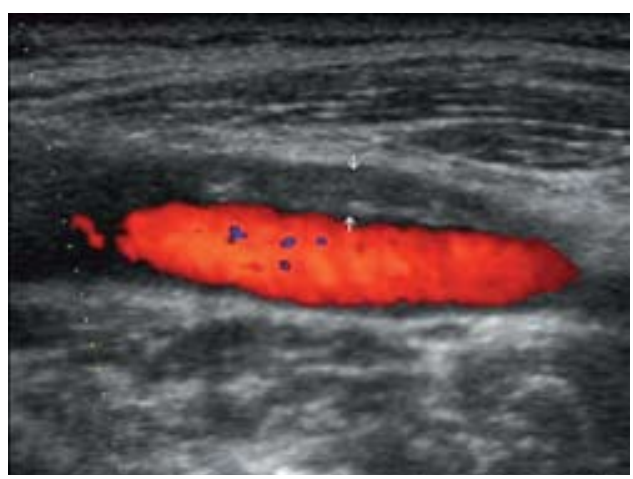

Figura 2c. Placa tipo III. Placa heterogénea predominatemente isoecoica-hiperecoica.

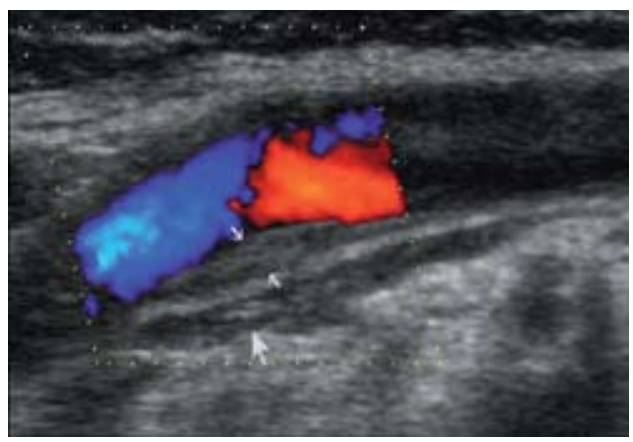

Figura 2d. Placa tipo IV. Placa homogénea isohiperecoica.

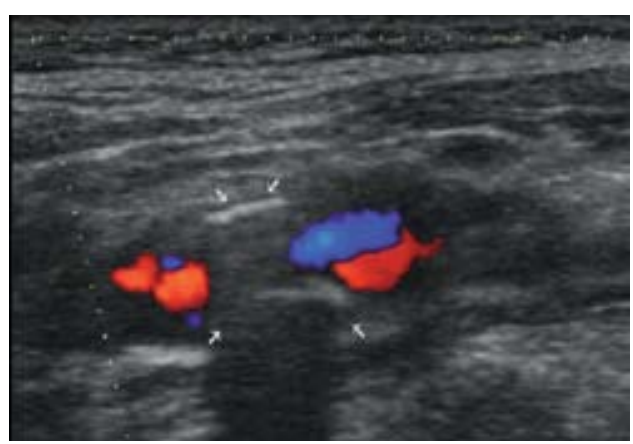

Figura 2e. Placa tipo V. Placa inclasificable (cálcica). 
- Índice de placa de ateroma (Índice aterogénico): Permite evaluar la magnitud de la ateroesclerosis en los sistemas carotídeos de acuerdo con lineamientos previamente descritos y validados ${ }^{(16)}$. El sistema carotídeo se divide en cuatro segmentos y para cada segmento el grado de placa se clasifica de la siguiente manera:

- Grado 0, sin placas

- Grado 1, una placa pequeña $(<30 \%$ del diámetro del vaso)

- Grado 2, una placa mediana (30 a 50\% del diámetro del vaso) o múltiples placas pequeñas.

- Grado 3, una placa grande (>50\% del diámetro de la arteria) o múltiples placas que incluyan al menos una placa mediana. Los grados de los cuatro segmentos de ambas carótidas se suman y se obtiene un índice de placa sensible para estimar la extensión de la ateroesclerosis. En nuestra investigación, clasificamos el índice aterogénico (IA) en 2 grupos: el primero incluyó todos aquellos pacientes que tuvieran un IA bajo menor de 1 (Grados 0 - 1) y en el segundo se ubicaron los que presentaron un IA aumentado, mayor de 1 (grados 2 y 3 ).

- Valoración hemodinámica de la estenosis carotídea (significativa o no): La estimación anatómica del porcentaje de luz arterial se hizo con cortes transversales (seleccionando el nivel en que la luz residual alcanza su menor diámetro). Se obtuvo el área residual y el área total de vaso, calculándose el porcentaje de estenosis en modo $B$ y se procedió a la toma de valores espectrales mediante Doppler pulsado, realizándose cortes longitudinales al vaso con una angulación 45 a $60^{\circ}$, lo más paralelo posible a éste. Se obtuvo las siguientes variables:

- Velocidad peak sistólica (VPS) de la arteria carótida interna $(\mathrm{ACl})$

- Velocidad diastólica final (VDF) de la arteria carótida interna $(\mathrm{ACl})$

- Velocidad peak sistólica (VPS) de la arteria carótida común (ACC)

Posteriormente se estimó la estenosis y se efectuó valoración morfológica según los parámetros reflejados por el Consenso de Estenosis Carotídea de la Sociedad Argentina de Cardiología y la Sociedad Neurológica Argentina. Revista Argentina de Cardiología / Vol. 74 nº 2 / marzo-abril 2006 (Tabla I).

Para el segundo estudio imaginológico se utilizó un dispositivo central Digital 2D Densitometer marca DMS Lexxos; el examen fue realizado siempre por el mismo operador, con 5 años de experiencia en su ejecución.

La densitometría ósea se efectuó con máquina $\operatorname{DEX} A^{(9,10)}$, ingresando los datos demográficos del paciente, tales como: la edad, el sexo, la raza, el peso y la talla. Para evitar factores técnicos pueden afectar los resultados, el equipo fue sometido a un riguroso control de calidad, realizándose calibraciones periódicas y barrido con fantomas. Se medió la DMO a nivel de la columna lumbar y caderas.

El radiólogo analizó e interpretó las imágenes, informando los resultados según la siguiente clasificación ${ }^{(11)}$ :

- Puntación o clasificación T: Los resultados de la DXA se comparan con la densidad ósea óptima de un adulto de 30 años de edad, del mismo género, en buen

Tabla I. Criterios ecográficos de estenosis carotídea (Consenso de Estenosis Carotídea Sociedad

Argentina de Cardiología y Neurología).

\begin{tabular}{|c|c|c|c|c|}
\hline \multirow{3}{*}{ Grado de estenosis } & \multicolumn{4}{|c|}{ Criterios } \\
\hline & \multirow{2}{*}{$\begin{array}{l}\text { VPS ACI } \\
(\mathrm{cm} / \mathrm{seg})\end{array}$} & \multicolumn{2}{|c|}{ Estimación de índice } & \multirow{2}{*}{$\begin{array}{l}\text { VDF ACI } \\
(\mathrm{cm} / \mathrm{seg})\end{array}$} \\
\hline & & $\begin{array}{l}\text { Placa en } \\
\text { modo B }\end{array}$ & $\begin{array}{l}\text { VPS ACI/ } \\
\text { VPS ACC }\end{array}$ & \\
\hline Normal & $<130$ & Sin placa & $<2$ & $<40$ \\
\hline$<50 \%$ & $<130$ & Placa $<50 \%$ & $<2$ & $<40$ \\
\hline $50 a<70 \%$ & $130-230$ & Placa $>50 \%$ & 2,0 a 4,0 & 40 a 100 \\
\hline$>=70 \%$ & $>230$ & Placa $>50 \%$ & $>4,0$ & $>100$ \\
\hline Crítica o preoclusiva & $\begin{array}{l}\text { Alta, baja o no } \\
\text { detectable }\end{array}$ & $\begin{array}{c}\text { Placa con luz } \\
\text { visible }\end{array}$ & Variable & Variable \\
\hline 100\% (oclusión) & No detectable & Luz no detectable & Ausente & Ausente \\
\hline
\end{tabular}


estado de salud y el paciente recibe una calificación $T$ ( $T$ score). Una calificación de 0 significa que su $\mathrm{DMO}$ es igual a la normal de un adulto joven sano. La diferencia entre su DMO y la de un adulto joven sano se mide en desviaciones estándar (DE); cuantas más DE por debajo de 0 , más baja es su densidad ósea y mayor su riesgo de fractura: se considera normal o saludable una calificación T entre +1 y -1 , densidad ósea baja entre 1.01 y 2.49 y osteoporosis si T es < de 2.5. Cuanto más grande es el número negativo, más severa es la osteoporosis.

Definiciones de la Organización Mundial de la Salud según los niveles de DMO (Tomados de: The World Health Organization Study Group. WHO Technical Report Series No.843. Geneva, 1994.):

- Normal: La DMO está dentro de 1 DE (+1 ó -1) del promedio para un adulto joven.

- Baja: La DMO está entre 1.01 y 2.49 DE por debajo del promedio para un adulto joven (-1.01 a -2.49 SD); indica osteopenia.

- Osteoporosis: La DMO está a 2.5 DE o más por debajo del promedio para un adulto joven (> -2.5 SD o más baja).

- Osteoporosis grave: La DMO está a más de 2.5 DE por debajo del promedio para un adulto joven y han ocurrido una o más fracturas producidas por la osteoporosis.

La información disponible ${ }^{(12-15)}$ no permite afirmar ni negar que los criterios actuales de la Organización Mundial de la Salud para el diagnóstico de la osteoporosis, que fueron diseñados para la mujer menopáusica, sean aplicables a la población masculina, por lo que en nuestro estudio decidimos utilizarlos sin considerar posibles diferencias relacionadas con el género. Tuvimos en cuenta, además, los resultados de una investigación realizada en 1.074 mujeres y 559 hombres sanos entre 20 a 70 años, durante 4 años
(1998 al 2002), en Ciudad de la Habana, donde no se hallaron diferencias.

- Puntación o Clasificación Z: La DMO del paciente se compara con la de un individuo típico de su misma edad. Esta comparación da la calificación Z. Como la DMO baja es común entre los adultos mayores, las comparaciones con la DMO de un individuo típico de su misma edad pueden ser engañosas. Es por esta razón que el diagnóstico de osteoporosis o de masa ósea baja se basa en la calificación T que se haya obtenido. Sin embargo, una calificación Z puede ser útil para determinar si hay una enfermedad subyacente o una condición que esté causando la pérdida de hueso.

Se utilizó la distribución de frecuencia calculando el porcentaje en variables cualitativas. En las variables cuantitativas se calculó la media aritmética y la DS. Se utilizaron además los siguientes procedimientos estadísticos bivariados:

- Se utilizó la prueba Chi-cuadrado para relacionar dos variables cuantitativas, con un nivel de significación del $5 \%,(\alpha=0,05)$.

- Se utilizó el coeficiente de Pearson para determinar posible correlación lineal entre dos variables cuantitativa con un nivel de significación del $5 \%,(\alpha=0,05)$.

\section{Resultados}

Estudiamos 115 pacientes con diagnóstico de ECVO, con edades comprendidas entre 42 y 85 años, con una media de 69,2 años, predominando el sexo masculino con un 62,6\% (Tabla II). El 46,9\% presentó osteopenia, el 29,6\% osteoporosis y el $23,5 \%$ una DMO normal, sin encontrar diferencias significativas en cuanto al sexo. Observamos mayor número de casos con osteopenia y osteoporosis en

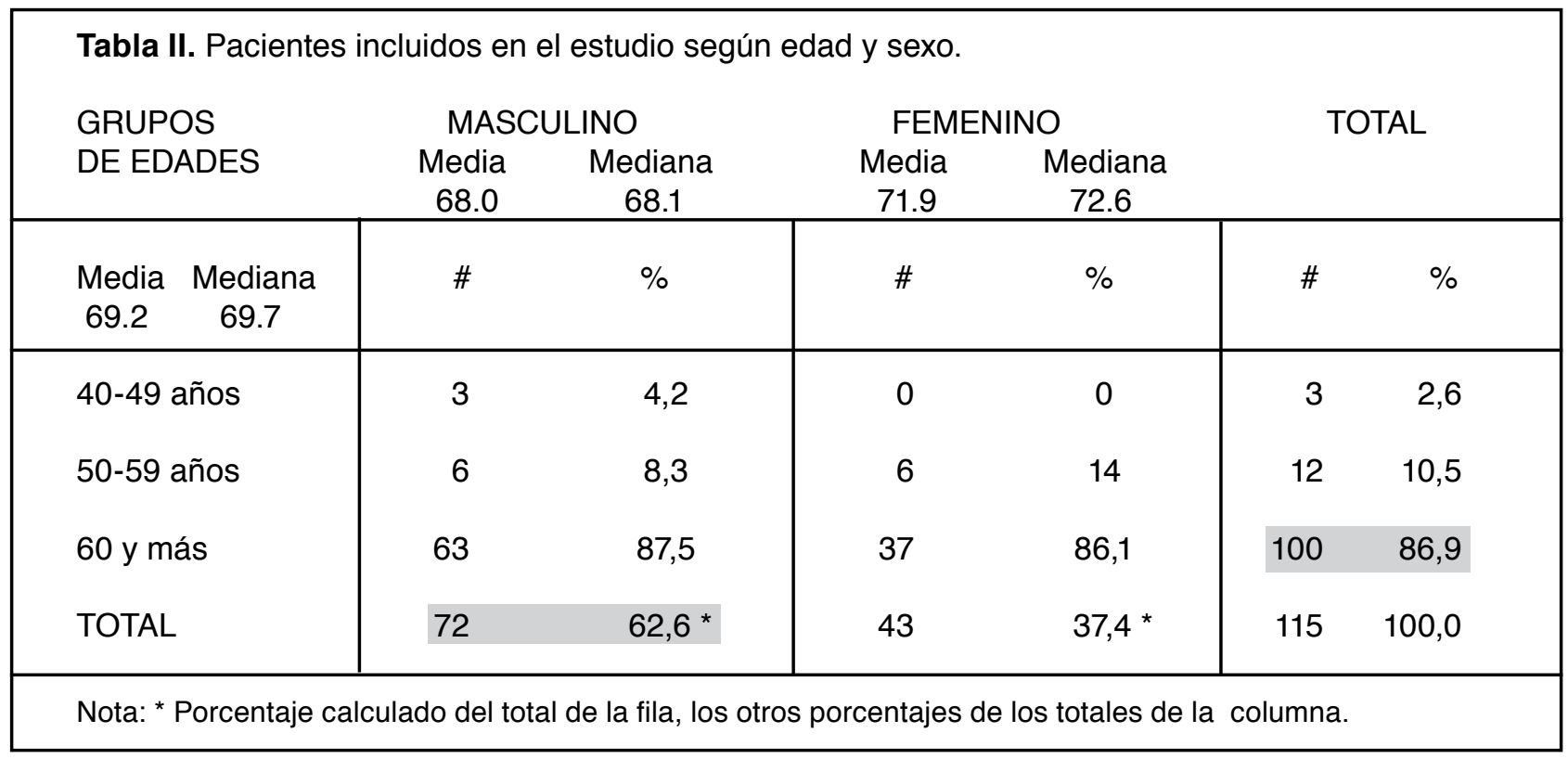


los pacientes mayores de 60 años (Tablas III y IV). En el comportamiento de la DMO según los factores de riesgo, se observó que todos ellos se asociaron a disminución de la DMO, a excepción de la DM, siendo el hábito de fumar el que tuvo mayor relación con el descenso de DMO (84.4\%). En general, la edad mayor de 60 años $(86,9 \%)$ y la HTA $(84,3 \%)$ fueron los factores de riesgo predominantes (Tabla V). Al examinar el comportamiento de la DMO según las diferentes manifestaciones ateroescleróticas estudiadas, observamos que la mayor parte de los pacientes con DMO normal tuvieron un IA mayor de 1 (66.4\%); sin embargo, la mayoría de los osteopénicos y osteoporóticos no tuvieron diferencias significativas en cuanto a los valores del IA. No encontramos asociación de valores de DMO con respecto al GIM y a las estenosis, pues en la mayoría de los casos predominó el GIM normal (51.3\%) y la estenosis no significativa (73.9\%), independientemente de los niveles de DMO (Tabla VI). El $93 \%$ de los pacientes presentaron lesiones estenooclusivas sin diferencias significativas en su influencia sobre la DMO. El tipo de placa predominantemente hiperecoica (tipo III) fue la de mayor frecuencia en el eje carotídeo, asociándose fundamentalmente con disminución de la DMO (Tabla VII). Al analizar la correlación de las variables aterogénicas: edad, índice de masa corporal (medidor de obesidad), IA (medidor de magnitud aterosclerótica) y GIM (medidor de lesión aterosclerótica precoz) con los niveles de DMO, sólo se demostró correlación del IMC con los valores de DMO en la columna y ambas caderas, con un nivel de significación estadística del 1\%, (moderadamente positivo); es decir, mientras mayor sea el IMC, mayores serán los valores de DMO y por tanto menor posibilidad de osteoporosis. El resto de los indicadores no presentaron asociación estadística con los valores de la DMO (Tabla VIII).

Tabla III. Pacientes según densidad mineral ósea y sexo.

\begin{tabular}{|c|c|c|c|c|c|c|}
\hline \multirow{2}{*}{$\begin{array}{l}\text { Densidad } \\
\text { mineral ósea }\end{array}$} & \multicolumn{2}{|c|}{ Masculino } & \multicolumn{2}{|c|}{ Femenino } & \multicolumn{2}{|c|}{ Total } \\
\hline & $\#$ & $\%$ & $\#$ & $\%$ & $\#$ & $\%$ \\
\hline Normal & 15 & 20.8 & 12 & 27.9 & 27 & 23.5 \\
\hline Osteopenia & 35 & 48.6 & 19 & 44.1 & 54 & 46.9 \\
\hline Osteoporosis & 22 & 30.5 & 12 & 27.9 & 34 & 29.6 \\
\hline Total & 72 & 100.0 & 43 & 100.0 & 115 & 100.0 \\
\hline
\end{tabular}

Tabla IV. Pacientes según densidad mineral ósea y edad.

\begin{tabular}{|c|c|c|c|c|c|c|c|c|}
\hline \multirow{2}{*}{$\begin{array}{l}\text { Grupos de } \\
\text { edad }\end{array}$} & \multicolumn{2}{|c|}{ Normal } & \multicolumn{2}{|c|}{ Osteopenia } & \multicolumn{2}{|c|}{ Osteoporosis } & \multicolumn{2}{|c|}{ Total } \\
\hline & $\#$ & $\%$ & $\#$ & $\%$ & $\#$ & $\%$ & \# & $\%$ \\
\hline 40 a 49 años & 1 & 3.8 & 1 & 1.8 & 1 & 2.9 & 3 & 2.6 \\
\hline 50 a 59 años & 4 & 14.8 & 5 & 9.3 & 3 & 8.8 & 12 & 10.5 \\
\hline 60 y más & 22 & 81.4 & 48 & 88.9 & 30 & 88.3 & 100 & 86.9 \\
\hline Total & 27 & 23.5 & 54 & 46.9 & 34 & 29.6 & 115 & 100 \\
\hline
\end{tabular}

Tabla V. Densidad mineral ósea según factores de riesgo.

\begin{tabular}{|c|c|c|c|c|c|c|}
\hline \multirow{3}{*}{ Factores de riesgo } & \multicolumn{4}{|c|}{ Densidad mineral ósea } & \multirow{2}{*}{\multicolumn{2}{|c|}{ Total }} \\
\hline & \multicolumn{2}{|c|}{ Normal } & \multicolumn{2}{|c|}{ Osteopenia y osteoporosis } & & \\
\hline & $\#$ & $\%$ & \# & $\%$ & $\#$ & $\%$ \\
\hline HTA & 25 & $25.7^{*}$ & 72 & $74.2^{\star}$ & 97 & 84.3 \\
\hline Edad > de 60 años & 22 & $22.0^{*}$ & 78 & $78.0^{\star}$ & 100 & 86.9 \\
\hline Diabetes mellitus & 15 & $51.7^{*}$ & 14 & $48.3^{\star}$ & 29 & 25.2 \\
\hline Cardiopatía isquémica & 8 & $24.2^{*}$ & 25 & $75.8^{\star}$ & 33 & 28.6 \\
\hline ECV anterior & 12 & $25.0^{\star}$ & 36 & $75.0^{\star}$ & 48 & 41.7 \\
\hline Hábito de fumar & 5 & $15.6^{\star}$ & 27 & $84.4^{\star}$ & 32 & 27.8 \\
\hline Alcoholismo & 6 & $25.0^{\star}$ & 18 & $75.0^{\star}$ & 24 & 20.8 \\
\hline Dislipidemia & 13 & $37.1^{\star}$ & 22 & $62.9^{\star}$ & 35 & 30.4 \\
\hline Obesidad & 16 & $44.4^{\star}$ & 20 & $55.6^{\star}$ & 36 & 31.3 \\
\hline
\end{tabular}


Tabla VI. Asociación entre las manifestaciones ateroescleróticas y osteoporosis.

\section{Manifestaciones ateroescleróticas}

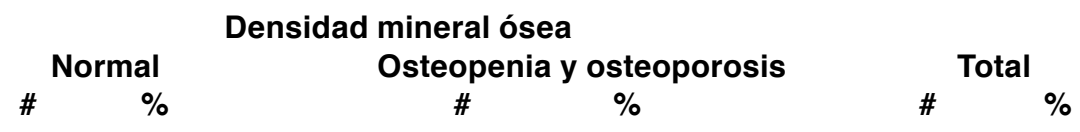

\begin{tabular}{|c|c|c|c|c|c|c|}
\hline \multicolumn{7}{|c|}{ Índice aterogénico } \\
\hline Menor de 1 & 9 & 33.4 & 46 & 52.3 & 60 & 52.2 \\
\hline Mayor de 1 & 18 & 66.6 & 42 & 47.7 & 55 & 47.8 \\
\hline Total & 27 & 100.0 & 88 & 100.0 & 115 & 100.0 \\
\hline \multicolumn{7}{|c|}{ GIM } \\
\hline Normal & 16 & 59.3 & 43 & 48.9 & 59 & 51.3 \\
\hline Engrosado & 11 & 40.7 & 45 & 51.1 & 56 & 48.7 \\
\hline Total & 27 & 100.0 & 88 & 100.0 & 115 & 100.0 \\
\hline \multicolumn{7}{|c|}{ Estenosis carotídea } \\
\hline No significativa & 19 & 70.4 & 66 & 75.0 & 85 & 73.9 \\
\hline Significativa & 8 & 29.6 & 22 & 25.0 & 30 & 26.1 \\
\hline Total & 27 & 100.0 & 88 & 100.0 & 115 & 100.0 \\
\hline
\end{tabular}

Tabla VII. Asociación entre DMO y tipo de placa ateromatosa predominante.

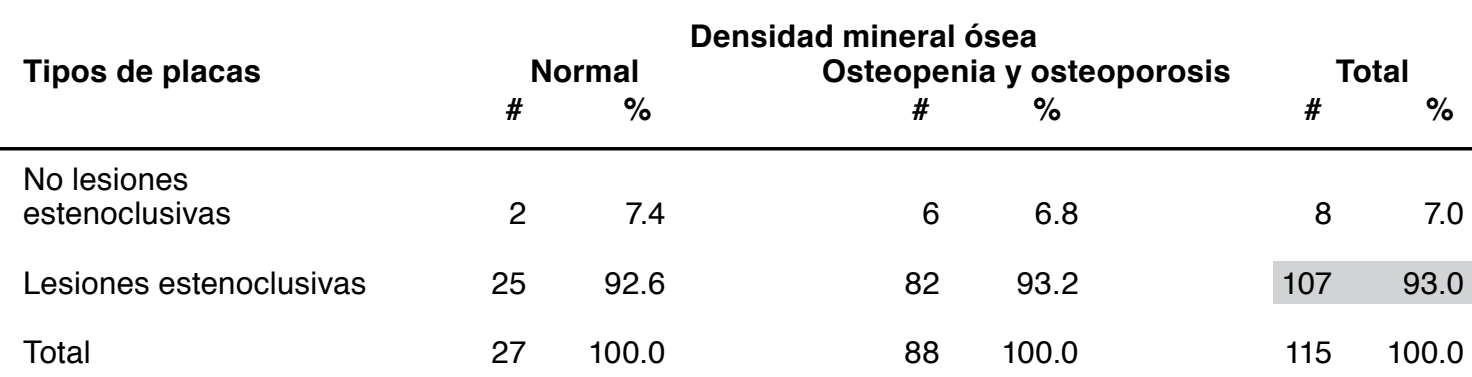

Predominantemente

iso-hiperecoica

$\begin{array}{rr}21 & 84.0 \\ 4 & 16.0 \\ 25 & 100.0\end{array}$

Tipo de lesiones oclusivas

Otros tipos de lesiones

Total

$25 \quad 100.0$

\begin{tabular}{rr}
72 & 87.8 \\
10 & 12.2 \\
\hline 82 & 100.0
\end{tabular}

$93 \quad 86.9$

$14 \quad 13.1$

$107 \quad 100.0$

Tabla VIII. Correlación de Pearson entre algunas variables y los niveles de DMO.

\begin{tabular}{|c|c|c|c|c|c|c|}
\hline \multirow{2}{*}{ Variables } & \multicolumn{2}{|c|}{ T Score columna } & \multicolumn{2}{|c|}{ T Score cadera der } & \multicolumn{2}{|c|}{ T Score cadera izq } \\
\hline & $\rho$ & $\mathrm{p}$ & $\rho$ & $\mathrm{p}$ & $\rho$ & $p$ \\
\hline Edad & 0.007 & 0.943 & -0.074 & 0.430 & -0.109 & 0.245 \\
\hline IA & 0.105 & 0.266 & 0.003 & 0.978 & -0.045 & 0.637 \\
\hline GIM & 0.010 & 0.918 & -0.105 & 0.266 & -0.060 & 0.523 \\
\hline IMC & $0.397^{\star *}$ & 0.010 & $0.341^{\star \star}$ & 0.010 & $0.454^{\star \star}$ & 0.010 \\
\hline
\end{tabular}




\section{Discusión}

La posible relación de la osteoporosis y la ateroesclerosis basada en la existencia de factores de riesgo en común ha sido ampliamente estudiada habiéndose encontrado resultados dispares, si bien a mayor número de factores de riesgo aumenta claramente la prevalencia de estas dos enfermedades tan comunes en la población adulta ${ }^{(16-18)}$.

Entre los factores de riesgo de la enfermedad ateroesclerótica que han sido relacionados con el metabolismo óseo están: HTA, dislipidemia y tabaquismo. Existen estudios epidemiológicos que han demostrado la asociación de la HTA ${ }^{(19)}$ con una baja DMO y también con incremento patológico del $\mathrm{GIM}^{(20,21)}$. Se sabe que la hiperlipidemia promueve la génesis de las placas de ateroma en las paredes arteriales al mismo tiempo que inhibe la diferenciación osteoblástica, con la consiguiente disminución de la $\mathrm{DMO}^{(22-25)}$. Yamaguchi et al(26) observaron relación inversa entre los niveles de colesterol LDL y la densidad ósea vertebral. Stulc et al ${ }^{(27)}$ encontró una correlación negativa entre la densidad ósea vertebral y el nivel de colesterol / triglicéridos sérico $(r=0.20-0.39, p<$ 0.05 ), corroborando la hipótesis de que la presencia de las lipoproteínas del plasma puede aumentar la isquemia del tejido y socavar el metabolismo óseo; sin embargo, otros trabajos no encuentran asociación entre los niveles de lípidos en sangre y la $\mathrm{DMO}^{(28-30)}$. El consumo de tabaco también es un conocido factor de riesgo para el desarrollo de osteoporosis y ateroesclerosis ${ }^{(16)}$ y se relaciona con una masa ósea baja ${ }^{(30,31)}$. Un metaanálisis publicado en el $2001^{(32)}$ analiza la DMO en fumadores de ambos sexos, llegando a la conclusión que tienen disminución de la DMO en caderas, columna, antebrazo y calcáneo respecto de los no fumadores; Broulik y Kapitola demostraron igual asociación ${ }^{(33)}$.

En nuestro estudio demostramos una mayor frecuencia de GIM patológico e índice aterogénico $\geq 1$ en los pacientes con consumo crónico de alcohol y una escasa correspondencia con la osteoporosis, si bien el mayor número de los pacientes con dicho hábito presentó bajos niveles de DMO. La ingesta de alcohol puede por sí sola puede provocar pérdida de masa ósea, osteomalacia u osteoporosis ${ }^{(34,35)}$, aunque se ha reportado que una ingesta moderada puede aumentar la masa ósea $^{(35-37)}$. El alcohol inhibe la proliferación de células osteoblásticas en cultivo ${ }^{(38)}$; su consumo prolongado y en exceso disminuye los metabolitos de la vitamina $D$ y causa hipogonadismo secundario, que es perjudicial para el hueso ${ }^{(34)}$. Respecto al desarrollo de enfermedad vascular, el consumo crónico de grandes cantidades de alcohol favorece el desarrollo de HTA ${ }^{(39)}$ y aumenta la incidencia de enfermedad vascular periférica ${ }^{(40)}$.

La obesidad se considera un factor de riesgo ateroesclerótico independiente, además de que los obesos tienen cifras más elevadas de tensión arterial, glicemia y colesterol( ${ }^{(4,42)}$. Contrariamente, la obesidad se asocia a mayor masa ósea, siendo un factor protector de osteoporosis $^{(43,45)}$. En nuestro estudio, la obesidad no tuvo una relación significativa con el daño ateroesclerótico.

El resto de los factores de riesgo estudiados no mostraron correlación con la osteoporosis, según lo esperado ${ }^{(46,47)}$, ni tampoco con la ateroesclerosis, al contrario de lo referido en la literatura ${ }^{(48,49)}$.

La asociación entre osteoporosis y arterioesclerosis está basada fundamentalmente en la existencia de estudios epidemiológicos que encuentran un aumento de la mortalidad por enfermedad cardiovascular en las personas con fracturas o con simple disminución de la DMO. La presencia de factores de riesgo en común, como: envejecimiento, depleción de estrógenos o consumo de tabaco parecen desempeñar un importante papel, pero también existen otros posibles mecanismos de vinculación patogénica menos conocidos que relacionan ambas enfermedades ${ }^{(5,50)}$.

Pennisi et al ${ }^{(51)}$ encontraron un mayor recambio óseo y reducción de las medidas de la masa ósea en pacientes con ateroesclerosis avanzada; todavía es incierto si la baja masa ósea resulta de una mayor calcificación arterial o viceversa o, si sólo comparten el mismo mecanismo fisiopatológico. La teoría de que hay migraciones de calcio de los huesos para el tejido vascular es señalada por algunos autores, siendo la desmineralización ósea directamente proporcional a la mineralización de la matriz colágena vascular ${ }^{(52,53)}$.

Diferentes investigaciones relacionan la presencia de enfermedad vascular (enfermedad arterial coronaria, ECV y enfermedad arterial periférica) con el desarrollo de osteoporosis ${ }^{(52,54-60)}$. Gupta y Aronow(55), revisando las historias clínicas de 102 mujeres posmenopáusicas, ponen de manifiesto que la enfermedad vascular aterosclerótica estuvo presente en el $51 \%$ de las mujeres con osteoporosis u osteopenia, medida por DEXA. $\mathrm{Ness}^{(54)}$ en un estudio retrospectivo en 1.000 mujeres postmenopáusicas, observó que la enfermedad vascular aterosclerótica era más comúnmente apreciada entre las mujeres con osteoporosis $(60 \%)$ que entre las osteopénicas $(35 \%)$ o normales $(22 \%)(p<0,001)$. La enfermedad vascular ateroesclerótica fue asimismo más prevalente en mujeres con osteopenia que en las que tenían niveles de DMO normal $(p<0,001)$. Por otra parte, en un grupo de mujeres se ha relacionado la gravedad de la placa arteroesclerótica carotídea (valorada mediante US), con la DMO total $\left(\mathrm{g} / \mathrm{cm}^{2}\right)$ determinada por DEXA ${ }^{(53)}$, determinándose que la DMO disminuye a medida que aumenta el número de placas. En este sentido, además se ha encontrado una significativa correlación positiva inversa entre el porcentaje de placas carotídeas hiperecogénicas y la osteoporosis $(p=0.016)$ en pacientes con enfermedad cerebrovascular, pudiendo constituir así la placa hiperecoica (tipo III) un marcador independiente de osteoporosis ${ }^{(56)}$. De igual manera, Tamaki ${ }^{(57)}$ y Sumino ${ }^{(59)}$ encontraron asociación entre la osteoporosis y la ateroesclerosis carotídea en los primeros 10 años después de la menopausia, al observar un incremento progresivo del GIM a medida que disminuía la DMO. La mayoría de estos trabajos han sido realizados en mujeres, siendo los estudios en varones más escasos. El trabajo de Gardsell et al(61) señala un aumento de mortalidad global en varones, con odds ratio (OR) de 1,4 por cada DE de descenso en la DMO de muñeca. Van der Klift et al(62) llegan a resultados similares, describiendo en varones una OR de 1,14 para mortalidad 
por todas las causas, por descenso en $1 \mathrm{DE}$ de la DMO en el cuello de fémur. En nuestra investigación no encontramos analogía con los datos reportados, pues el GIM, la estenosis y el IA no mostraron asociación con los valores de la DMO e incluso la mayor parte de los pacientes con DMO normal tuvieron un IA aumentado y la mayoría de los osteopénicos lo presentaron normal. Con relación al tipo de placa, predominó la hiperecoica independientemente de los valores de la DMO. Además, no encontramos correlación significativa con la edad, el GIM (lesión aterosclerótica precoz) y el IA (magnitud aterosclerótica); se observó solamente con el IMC, siendo moderadamente positiva (nivel de significación $=1 \%$ ), lo que sí coincide con lo referido por casi todos los autores que han hecho alusión al tema, quienes plantean que el sobrepeso y la obesidad se asocian a mayor masa ósea, factor protector de osteoporosis ${ }^{(16)}$. Sin embargo, la mayor parte de nuestros obesos presentó un DMO disminuida, que pudo estar en relación a posibles cambios bruscos de peso como ha sido sugerido en varios estudios $^{(41,42,60)}$

Algunos autores han encontrado resultados similares en cuanto al hecho de no demostrar asociación entre osteoporosis y ateroesclerosis, sobre todo en lo que se refiere a calcificación aórtica, coronaria y placas de ateroma, pero no a enfermedad cerebrovascular. Por ejemplo, un trabajo sobre calcificación aórtica y DMO a nivel lumbar medida por densitometría en 130 mujeres postmenopáusicas, no muestra una asociación significativa ${ }^{(63)}$ y otro trabajo realizado por Vogt et al ${ }^{(64)}$, en 2.051 mujeres mayores de 65 años tampoco muestra asociación entre DMO y el grado de calcificación de la aorta, por lo que estos autores proponen que simplemente ambos procesos, osteoporosis y calcificación aórtica, son independientes y acompañan al envejecimiento sin relación causal. De esta misma opinión son Aoyagi et al, 65 que no encuentran asociación entre la calcificación de la aorta y la DMO (índice Z) en 524 mujeres japonesas tras ajustar por edad, tensión arterial sistólica, actividad física y tabaco. Sinnot ${ }^{(66)}$, en 313 mujeres posmenopáusicas y 167 hombres sugiere, luego de ajustar por edad, que la osteoporosis y la ateroesclerosis coronaria son procesos independientes y Hmamouchi(67) tampoco encontró relación entre la osteoporosis y presencia de placas carotídeas o femorales en 54 pacientes menopáusicas marroquíes $(p=0,271)$.

Nuestra investigación se basa en una serie sesgada que no incluyó grupo control, lo que pudo influir en los resultados obtenidos. En todos los casos, el US Doppler fue realizado por el mismo especialista con experiencia de 12 años en la realización de dicho examen, evitando así la variabilidad interoperadores; lo mismo ocurrió con la densitometría que siempre fue hecha por el mismo operador con 5 años de práctica en su ejecución. Sin embargo, nuestro estudio pudo estar sesgado al no medir la variabilidad intraoperador.

\section{Conclusiones}

No encontramos asociación entre la osteoporosis y las manifestaciones ateroescleróticas carotídeas diagnosticadas mediante US Doppler color y densitometría, en los pacientes con ECVO. Tampoco se obtuvo correlación entre el valor de la DMO y la magnitud del daño aterosclerótico a nivel carotídeo. Sólo el IMC mostró una correlación moderada y positiva con los niveles de DMO, por lo que mientras mayor es el IMC, menor es el riesgo de osteoporosis. Nuestros hallazgos indican que, en la ECVO, la osteoporosis y las manifestaciones ateroescleróticas carotídeas no están relacionadas, más allá de la presencia de factores de riesgo en común.

\section{Bibliografía}

1. Sálica D. Arteriosclerosis y osteoporosis. 2006. Disponible en: URL: http://www.medwave.cl/congresos/ Osteologia2006/2/1.act.

2. Nowicka G, Panczenko-Kresowska B. Coronary heart disease and osteoporosis: factors related to development of both diseases. 2007. Disponible en: URL: http://www. ncbi.nlm.nih.gov/pubmed/17941467?ordinalpos=1\&itool= EntrezSystem2.PEntrez.Pubmed.Pubmed_ResultsPanel. Pubmed_DiscoveryPanel.Pubmed_Discovery_RA\&link pos $=4 \& \log \$=$ relatedarticles\&dbfrom $=$ pubmed.

3. Baldini V, Mastropasqua M, Francucci CM, D'Erasmo E. Cardiovascular disease and osteoporosis. Rev J Endocrinol Invest 2005; 28(10): 69-72.

4. Osteoporosis y ateroesclerosis comparten la fisiopatología. 2006. Disponible en: URL: http://www.diariomedico.com/ edicion/diario_medico/especialidades/reumatologia/es/ desarrollo/714891.html.

5. Hamerman D. Osteoporosis and atherosclerosis: biological linkages and the emergence of dual-purpose therapies. Rev QJM 2005; 98(7): 467-84.

6. Vemmos KN, Bots ML, Tsibouris PK, Zis VP, Takis CE, Grobbee DE, et al. Prognosis of stroke in the south of Greece: 1 year mortality, functional outcome and its determinants: the Arcadia Stroke Registry. J Neurol Neurosurg Psychiatry 2000; 69(5): 595-600.

7. Grant EG, Bemson CB, Moneta GL, Alexandrv AV, Baker JD, Bluth El, et al. Carotid Artery Stenosis: Gray-Scale and Doppler US Diagnosis. Society of Radiologists in Ultrasound. Consensus Conference. Radiology 2003; 229: 340-346

8. Martínez-Vila E, Irimia P. Factores de riesgo del ictus. Eur J Vasc Endovasc Surg; 2004; 14: 439-445.

9. Kanis JA, Glauer CC. An update on the diagnosis and assessment of osteoporosis with densitometry. Committee of Scientific Advisors, International Osteoporosis Foundation. Osteoporos Int 2000; 11: 192-202.

10. Montero M, Calabuig E, Muñoz ML, Valero Sanz JL, Todolí JR, Calabuig JR, et al. Densitometría axial y periférica en el diagnóstico de la osteoporosis. Rev Española de Enfermedades Metabólicas Óseas 2002; 11(4): 140-143.

11. Gómez de Tejada MJ, Sosa Henríquez M. "Los ultrasonidos, la densitometría, el T-SCORE y los criterios de la OMS para el diagnóstico de la osteoporosis". Rev Española de Enfermedades Metabólicas Óseas 2002; 11(5): 165-166.

12. Santos Hernández MC, Ugarte JC, González de la Nuez J. Criterios normativos de diagnóstico de la osteoporosis y la composición corporal en la población cubana. 2004. Disponible en: URL: http://www.monografias.com/ trabajos16/criterios-osteoporosis/criterios-osteoporosis. shtml.

13. Melton III LJ, Atkinson EJ, O Connor MK, O Fallon WM, BL Riggs. Bone density and fracture risk in men. J. Bone Miner Res 1998; 13: 1915-1923.

14. Melton III LJ, ES Orwoll, RD Wasnich. Does bone den- 
sity predict fractures comparably in men and women? Osteoporos Int. 2001; 12: 707-709.

15. Cheng S, Suominen H, Sakari-Kantala R, Laukkanen P, Avikainen V, Heikkinen E. Calcaneal bone mineral density predicts fracture occurrence. A five years follow up study in elderly people. J Bone Miner Res 1997; 12: 1075-1082.

16. Juan Más T, Cabanesi B. Prevalencia de osteoporosis y su asociación a factores de riesgo en personas mayores de ambos sexos en Mallorca. Medicina Balear 2007; 22(3).

17. González López A. Asociación entre factores de riesgos y ateroesclerosis carotídea en la enfermedad cerebrovascular oclusiva evaluadas por ecodoppler color. [Tesis de maestría]. Hospital Carlos J. Finlay 2008.

18. Dargent Molina P, Douchin MN, Cornier C, Meunier PJ, Breat G. EPIDOS Study Group. Use of clinical risk factors in elderly women with low bone mineral density to identify women at higher risk of hip fracture: The EPIDOS prospective study. Osteoporosis Int 2002; 13: 593-599.

19. Tsuda K, Nishio I, Masuyama Y. Bone mineral density in women with essential hypertension. Am J Hypertens 2001: 14: 704-707.

20. Zambón $\mathrm{D}$. Valoración de la placa aterogénica mediante ecografía arterial de carótidas. Importancia clínica y actitud terapéutica. JANO 2004; 66: 1518.

21. Poblete R. Marcadores precoces de ateroesclerosis. Técnicas diagnósticas. Rev Chilena de Cirugía 2005; 57(2): 101-108.

22. Parhami F, Garfinkel A, Demer LL. Role of lipids in osteoporosis. Arterioscler Thromb Vasc Biol 2000; 20: 2346-2348.

23. Mody N, Parhami F, Sarafian TA, Demer LL. Oxidative stress modulates osteoblastic differentiation of vascular and bone cells. Free Radic Biol Med. 2001; 31(4): 50919.

24. Parhami F. Posible role of oxidized lipidis in osteoporosis: could hyperlipidemia be a risk factor? Prostaglandins Leukot Essent Fatty Acids 2003; 68(6): 373-8.

25. Tintut $Y$, Morony S, Demer LL. Hyperlipidemia promotes osteoclastic potential of bone marrow cells ex vivo. Arterioscler Thromb Vasc Biol 2004; 24: 6-10.

26. Yamagushi T, Sugimoto T, Yano S, Yamauchi M, Sowa $H$, Chen Q, et al. Plasma lipids and osteoporosis in postmenopausal women. Endocr J 2002; 49: 211-217.

27. Stulc T, Ceska R, Horinek A, Stepan J. Bone mineral density in patients with apolipoprotein $E$ type 2/2 and 4/4 genotype. Physiol Res 2000; 49 (4): 435-9.

28. Solomon DH, Avorn J, Canning CF, Wang PS. Lipids levels and bone mineral density. Am J Med. 2005; 118: 1414.

29. Bagger $Y Z$, Rasmussen HB, Alexandersen P, Werge T, Christiansen C, Tankó LB. PERF study group. Links between cardiovascular disease and osteoporosis in postmenopausal women: serum lipids or atherosclerosis per se?. Osteoporos Int 2007; 18(4): 505-12.

30. Valtola A, Honkanen R, Kroger H, Tuppurainen M, Saarikoski S, Alhaya E. Lifestyle and other factors predict ankle fractures in perimenopausal women: a population- based prospective cohort study. Bone 2002; 30: 238-42.

31. Rapuri PB, Gallagher JC, Balhorn KE, Ryschon KL. Smoking and bone metabolism in elderly women. Bone 2000; 27: 429-36.

32. Ward KD, Klesges RC. A meta-analysis of the effects of cigarette smoking on bone mineral density. Calcif Tissue Int 2001; 68: 259-70.

33. Broulik PD, Kapitola J. Interrelations between body weight, cigarette smoking ans spine mineral density in osteoporotic
Czech women. Endocr Regul 1993; 27: 57-60.

34. Turner RT. Skeletal response to alcohol. Alcohol Clin Exp Res 2000; 24: 1693-701.

35. Cawthon PM, Harrison SL, Barrett-Connor E, Fink HA, Cauley $\mathrm{JA}$, Lewis CE, et al. Alcohol intake and its relationship with bone mineral density, falls, andfracture risk in older men. $J$ Am Geriatr Soc 2006 Nov; 54 (11): 1649-57.

36. Mukamal KJ, Conigrave KM. Roles of drinking pattern and type of alcohol consumed in coronary heart disease in men. N Engl J Med 2003; 348: 109-18.

37. Feskanich D, Korrick SA, Greenspan SL. Moderate alcohol comsumption and bone density among postmenopausal women. J Womens Health 1999; 8: 65-73.

38. Klein RF, Fausti KA, Carlos AS. Ethanol inhibits human osteoblastic cell proliferation. Alcohol Clin Exp Res 1996; 20: $572-8$.

39. Ganny O, Baudoin C, Fardellone P. Effect of alcohol intake on bone mineral dendity in elderly women: The EPIDOS Study. Epidemiologie de l'osteoporose. Am J Epidemiol 2000; 151: 773-80

40. Jepson RG, Fowkes FG, Donnan PT. Alcohol intake as a risk factor for peripheral arterial disease in the general population in the Edingburgh Artery Study. Eur J Epidemiol 1995; 11: 9-14.

41. Ravn P, Cizza G, Biamason NH. Low body mass index is an important risk factor for low bone mass and increased bone loss in early postmenopausal women. J Bone Miner Res 1999; 14: 1622-1627.

42. Wildner M, Peters A, Raghuvanshi VS. Superiority of age and weight as variables in predicting osteoporosis in postmenopausal white women. Osteoporosis Int 2003; 14: 950-956.

43. Joint British Societies'guidelines on prevention of cardiovascular disease in clinical practice. British Cardiac Society. British Hypertension Society, Diabetes, UK, Heart UK, Primary Care Cardiovascular Society, the Stroke Association. Heart 2005; 91 (V): 1-52.

44. Goldstein LB, Adams R, Alberts MJ, Appel LJ, Brass LM. Primary prevention of schemic stroke. AHA/ ASA Guideline Circ 2006; 113: 873-923.

45. Ivanova Georgieva R. Factores de riesgo cardiovascular y tratamiento hipolipemiante en la ECV, cardíaca y periférica. [Tesis doctoral]. Universidad de Granada 2007.

46. Danilevicious CF, Lopes JB, Pereira RMR. Bone metabolism and vascular calcification. Braz J Med Biol Res 2007; 40(4): 435-442.

47. González López A. Valor del ultrasonido Doppler-Duplex para evaluar el engrosamiento del endotelio vascular en relación a factores de riesgo de la ateroesclerosis. [Tesis de especialidad]. Hospital Carlos J. Finlay 2002.

48. Gonzáles-Macias J, Mann F, Vila J. Prevalencia de factores de riesgo de osteoporosis y fracturas osteoporóticas en una serie de 5195 mujeres mayores de 65 años. Med Clin (Barc) 2004; 12(3): 85-89.

49. Oliva JE, Enríquez LE, Cusa R, Canetti MA. Fernández Britto JE. Enfermedad cerebrovascular: comportamiento en el Hospital Docente "Dr. Salvador Allende" durante 1997. Rev Cubana Invest Bioméd. Ciudad de la Habana 2001; 20(3).

50. Pennisi P, Signorelli SS, Riccobene S. Low bone density and abnormal bone turnover in patients with atherosclerosis of peripheral vessels. Osteoporosis Int 2004; 15(5): 389-95.

51. Uyama O, Yoshimoto Y, Yamamoto Y, Kawai A. Bone changes and carotid atherosclerosis in postmenopausal women. Stroke 1997; 28: 1730-2.

52. Reddy J, Bilezikian JP, Smith SJ. Reduced bone mineral 
density is associated with breast arterial calcification. $J$ 52. Clin Endocrinol Metab 2008; 93(1): 208-211.

53. Valero Díaz de Lamadrid C, González Macias J. Osteoporosis y arteriosclerosis. Rev Esp Enf Metabolicas Óseas 2004, 13 (02): 34-45.

54. Ness J, Aronow WS. Comparison of prevalence of atherosclerotic vascular disease in postmenipausal women with osteoporosis or osteopenia versus without osteoporosis or osteopenia. Am J Cardiol.2006; 97(10): 1427-8.

55. Gupta G, Aronow W. Doença vascular aterosclerótica pode estar associada à osteoporose ou osteopenia na pós-menopausa. Revista científica de Gerontología y Geriatría Septiembre/octubre 2006.

56. Saverino A, Del Sette M, Conti M. Hyperechoic plaque: an ultrasound marker for osteoporosis in acute stroke patients with carotid disease. Eur Neurol 2006; 55(1): 31-6.

57. Tamaki J, Hirano Y, Sato Y. Low bone mass is associated with carotid ateroesclerosis in postmenopausal women: The Japanese Population-based Osteoporosis (JPOS) Cohort Study. Osteoporos Int 2008.

58. Tankó LB, Christiansen C, Cox DA, Geiger MJ, McNabb MA, Cummings SR. Relationship between osteoporosis and cardiovascular disease in postmenopausal women. J Bone Miner Res 2006; 21(2): 352.

59. Sumino H, Ichikawa S, Kasama S, Takahashi T, Sakamoto $\mathrm{H}$, Kumakura $\mathrm{H}$, et al. Relationship between carotid atherosclerosis and lumbar spine bone mineral density in postmenopausal women. Hypertens Res 2008; 31(6): 1191-7.
60. Hsu YH, Venners SA, Terwedow HA, Feng Y, Niu T, Li Z, et al. Relation of body composition, fat mass, and serum lipids to osteoporotic fractures and bone mineral density in Chinese men and women. Am J Clin Nutr 2006; 83(1): 146-54.

61. Gardsell P, Johnell O. Bone mass a marker of biologic age?. Clin Orthop 1993; 287: 90-3.

62. Van der Klift M, Pols HA, Geleijnse JM, Van der Kuip DA, Hofman A, De Laet. Bone mineral density and mortality in elderly men and women: The Rotterdam study. Bone 2002; 30:643-8.

63. Jorgensen L, Engstad T, Jacobsen BK. Bone mineral density in acute stroke patients: low bone mineral density may predict first stroke in women. Stroke 2001; 32: 47-51.

64. Vogt MT, San Valentin R, Forrest KY, Nevitt MC, Cauley JA. Bone mineral density and aortic calcification: the study of osteoporotic fractures. J Am Geriatr Soc 1997; 45: $140-5$.

65. Aoyagi K, Ross PD, Orloff J, Davis JW, Katagiri H, Wasnich RD. Low bone density is not associated with aortic calcification. Calcif Tissue Int 2001; 69: 20-4.

66. Sinnot B, Syed I, Sevrokov A. Coronary calcification and osteoporosis in men and posmenopausal women are independent processes associated with aging. Calcif Tissue Int 2006 Apr, 78(4): 195-202.

67. Hmamouchi F, Allali H, Mâaroufi M, Cherkaoui R, Abouqal $\mathrm{N}$. Relation entre ostéoporose et athérosclérose chez les patientes ménopausées marocaines. Société Française de Rhumatologie. Soumission Congrès 2006. 